\title{
Analyse de la Rentabilité Économique du Maraîchage d'hivernage dans les Communes d'Imanan et de Tagazar au Niger
}

\author{
Idrissa Guisso Maïga Djibril \\ Soumana Boubacar
}

Faculté d'Agronomie de l'Université Abdou Moumouni de Niamey, Niger

Doi:10.19044/esj.2021.v17n17p362

Submitted: 19 March 2021

Accepted: 01 May 2021

Published: 31 May 2021
Copyright 2021 Author(s)

Under Creative Commons BY-NC-ND

4.0 OPEN ACCESS

Cite As:

Maïga Djibril I.G. \& Boubacar S. (2021). Analyse de la Rentabilité Économique du Maraîchage d'hivernage dans les Communes d'Imanan et de Tagazar au Niger. European Scientific Journal, ESJ, 17(17), 362. https://doi.org/10.19044/esj.2021.v17n17p362

\section{Résumé}

Dans leur quête de sécurité monétaire, les ménages s'adonnent au maraîchage d'hivernage qui de nos jours, représente une véritable alternative de diversification de revenu. La présente étude analyse aussi bien la rentabilité économique des exploitations maraîchères d'hivernage que les facteurs socioéconomiques et techniques influençant cette rentabilité dans les communes d'Imanan et de Tagazar au Niger. Les données primaires ont été obtenues au moyen d'enquêtes individuelles et des focus groups. Au total, 102 maraîchers, choisis de façon aléatoire, ont été enquêtés. L'analyse des données basée sur l'approche budgétaire, a permis d'évaluer les éléments comptables des exploitations. Une fonction stochastique de type Cobb-Douglass a été estimée à l'aide du logiciel STATA 14.1. Les résultats montrent que le modèle est hautement significatif $(p<0,001)$ avec un coefficient de détermination multiple ajusté $\mathrm{R}^{2}$ de 0,79 . Il résulte que les exploitations maraîchères d'hivernage étudiées sont rentables, étant donné qu'un franc investi rapporte 3,47 FCFA. En ce qui concerne les déterminants, les résultats montrent que l'expérience des maraîchers, la superficie cultivée et la vente individuelle au marché ont un effet positif significatif sur la marge nette dégagée. Pendant que la vente groupée bord parcelle l'influence négativement. Le maraîchage d'hivernage doit être davantage promu au Niger tout en mettant l'accent sur la vente individuelle au marché. 
Mots clés : Exploitations maraîchères d'hivernage, Rentabilité, Marge nette, Déterminants, Niger

\title{
Analyzing Economic Profitability of Rainy Market Garden in the Municipalities of Imanan and Tagazar in Niger
}

\section{Idrissa Guisso Maïga Djibril \\ Soumana Boubacar}

Faculté d'Agronomie de l'Université Abdou Moumouni de Niamey, Niger

\begin{abstract}
In their quest for monetary security, the households practice the rainy market gardening which is, at present, a real alternative of income diversification. This paper focuses on the rainy market gardening profitability in the municipalities of Imanan and Tagazar in Niger. It aims to identify the factors which explain this profitability. The data collected in the fields is used to calculate the different inputs, output, and the profitability of rainy market gardening. Cobb Douglass stochastic function is estimated. The model is significative $(\mathrm{p}<0,001)$ with an adjusted coefficient of determination $\mathrm{R}^{2}$ of 0,79 . The model explains about $79 \%$ of net margin changing by the variables included. The result shows that on an average area of $180 \mathrm{~m}^{2}$, a farmer who invests 74000 FCFA in rainy market garden earns a net margin of 177156 FCFA. After using the regression model, it reveals that the net margin of rainy market gardening is influenced by variables like the experience, the area of gardening, and the selling product at the market. However, the age, being in famer's organization, access to information and other selling strategies were not significative in the model.
\end{abstract}

Keywords: Rainy gardening market, Profitability, Net margin, Determinants, Niger

\section{Introduction}

Les potentialités dont dispose le Niger en termes de terres arables, d'eau souterraine et de ressources humaines font de l'agriculture le moteur de l'économie de ce pays (INS, 2012). Malgré son importance, l'agriculture est soumise à de nombreuses contraintes impactant négativement sa performance. Ainsi observe-t-on très peu d'améliorations dans les pratiques culturales ancestrales basées surtout sur la production de cultures sèches mais qui apparaissent de plus en plus limitées pour faire face efficacement aux rapides changements socio-économiques et climatiques actuels (Butaré \& Zoundi, 
2005). La pratique de la jachère, garante de la restauration naturelle de la fertilité des sols est de nos jours sous la pression foncière et d'un besoin galopant en résidus de culture, menacée de disparition (CILSS, 1982a; OCDE/FAO, 2016). Les attaques parasitaires, notamment le développement des acridiens et des chenilles se manifeste de plus en plus du fait de la prédominance de la monoculture basée sur les céréales (CILSS, 1982b ; Forest et $a l ., 1991$; Balme et al., 2005). Il en résulte une baisse continue de la productivité agricole en déphasage avec un taux de croissante de la population ascendant (Hamadou, 2000). Pour pallier aux difficultés des paysans liées à la dégradation de leur revenu dont la faible valeur marchande de leur production céréalière ne permet plus de garantir, mais face aussi aux effets pervers des aléas climatiques, des initiatives proposées par l'Etat et soutenues par les partenaires au développement ont été introduites en milieu rural. Il s'agit notamment de la culture maraichère de contre-saison.

Pratiquées pour fournir des compléments alimentaires, les cultures de contre saison ont été très vite appropriées par les populations, à telle enseigne que la valeur d'échange est supérieure à la valeur d'usage. Cette situation a occasionné, au fil du temps, un important volume de productions maraîchères sur une courte période. La conséquence est la baisse des prix des produits maraîchers obligeant certains producteurs à brader leurs extrants (Mahamadou et $a l ., 2018)$. Face à cette nouvelle situation, des producteurs entreprenants, en retardant la production pour bénéficier de prix rémunérateurs, ont fini par identifier une forte demande en produits frais notamment en hivernage. Etant dans une perte de confiance quant à l'investissement dans les cultures sèches, pratiquer une activité à haute valeur marchande mais aussi d'usage, dont ils ont une marge dans la maitrise de l'eau et de certains intrants, est devenu une aubaine pour les producteurs ayant déjà une expérience dans les cultures de contre saison. Ce constat est surtout fait de la périphérie de Niamey jusqu'à un rayon de $100 \mathrm{~km}$ notamment dans les zones dont l'accès à l'eau souterraine est plus facile telles que les communes de Tagazar et d'Imanan (Nazoumou et al., 2016). Les deux communes toutes traversées par le Dallol Bosso, se particularisent dans la production maraîchère d'hivernage. La première est spécialisée dans la production du chou tandis que la seconde contribue dans la production de la tomate et du poivron. L'objectif de ce travail est d'analyser la rentabilité économique des exploitations maraîchères d'hivernage tout en déterminant les facteurs explicatifs de cette dernière. Une exploitation maraîchère d'hivernage peut être définie comme étant une entité technicoéconomique orientée dans la production des légumes et des fruits durant la campagne humide. Cette campagne peut s'étendre du mois de mai à octobre (MAG/El, 2015). 


\section{Matériel et méthodes}

\subsection{Zone d'étude}

Les communes de Tagazar et d'Imanan sont situées respectivement, à $13^{\circ} 47^{\prime} 7^{\prime \prime}$ 'Nord et $02^{\circ} 56^{\prime} 50^{\prime \prime}$ 'Est et $14^{\circ} 01^{\prime} 40^{\prime \prime}$ 'Nord et $3^{\circ} 12^{\prime} 53^{\prime \prime}$ Est, dans les départements de Balleyara et de Filingué. Traversées par le Dallol Bosso (une vallée fossile), les deux (2) communes bénéficient d'une nappe peu profonde et d'une proximité avec Niamey, la capitale.

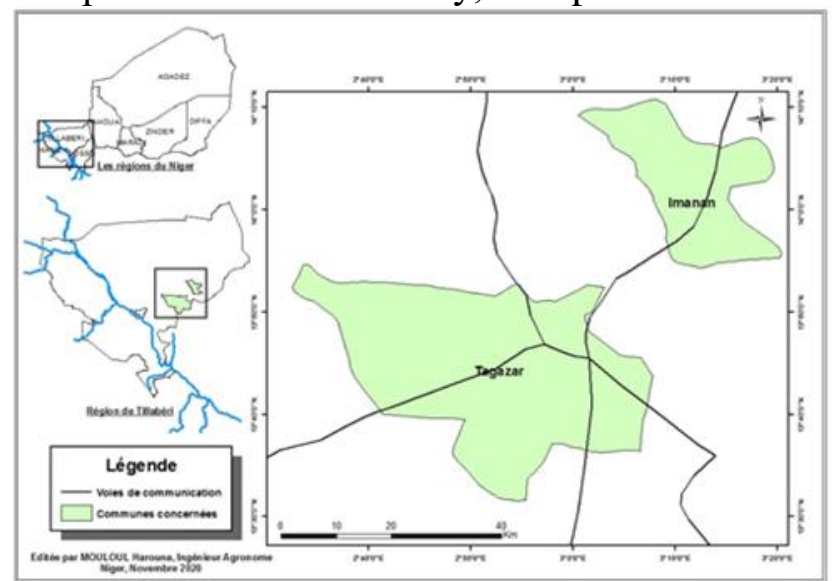

Figure 1. Zone d'étude

\subsection{Matériel}

Le matériel végétal est constitué de chou (Brassia oleracea), de poivron (Capsicum annuum) et de tomate (Solanum lycopersicum), qui sont les principales spéculations dans les parcelles. Le matériel technique de collecte de données comprend un guide d'entretien semi-structuré, un questionnaire et un appareil GPS.

\subsection{Méthodes}

\subsubsection{Présentation des données}

Les données secondaires ont été recueillies auprès des services techniques, municipaux et les bibliothèques pour obtenir des informations techniques et d'ordre général de la zone d'étude. Ces données nous ont permis de faire l'état de lieu des concepts et théories se rapportant à notre recherche. Les données primaires ont été obtenues au moyen d'enquêtes individuelles et des focus groups. Les valeurs des extrants récoltés et des intrants utilisés ont été quantifiées afin de déterminer les ratios économiques.

\subsubsection{Critères et choix des sites}

La pratique du maraîchage toute l'année, l'accès rapide aux grands centres et l'accès facile à l'eau constituent les trois principaux critères ayant prévalu dans le choix de la zone d'étude. L'échantillonnage a été réalisé à la 
suite de deux étapes. Il s'agit d'abord, de la prise contact avec les services techniques et les leaders paysans. Le concours de ces deux acteurs agricoles expérimentés connaissant bien la zone, a permis d'évaluer le nombre de maraîchers d'hivernage à 450 en 2019 dans les communes d'Imanan et de Tagazar. L'objectif fixé étant d'enquêter au moins $20 \%$ de cette population cible, la collecte de données a concerné 102 maraîchers d'hivernage.

\subsubsection{Détermination de la rentabilité}

Plusieurs auteurs ont essayé de définir la rentabilité et les méthodes de son évaluation (Babacar et al., 2020). Son calcul diffère selon les aspects économiques, commerciaux et financiers (Khadija \& Soukaina, 2011). Selon Pirou (2005), la rentabilité est la capacité d'un capital à obtenir un revenu. On compare le profit obtenu au capital engagé. La rentabilité peut se définir aussi comme étant la capacité d'une entreprise à réaliser des bénéfices sur une période donnée. Pour Beiton et Ali (2001), la rentabilité est un rapport entre les gains et les fonds engagés. Elle mesure l'aptitude d'une opération économique à produire un flux de revenus actualisés supérieurs aux dépenses engagées. Elle s'exprime par un taux qui ne tient pas compte de l'origine des capitaux engagés dans l'opération et est mesurée par la valeur ajoutée. Selon l'institut national des statistiques et études économiques (INSEE), la marge brute qui est le solde du compte de production est égale à la valeur de la production diminuée des consommations intermédiaires. Selon cette même source, l'approche budget d'entreprise est mieux indiquée pour ressortir les éléments comptables des exploitations. Ainsi, les différentes formules utilisées résultent de cette revue littéraire.

-La marge brute (MB) est formulée comme suit:

MB = Produit brut - Charges Opérationnelles

Les charges opérationnelles constituent les montants alloués à l'achat des semences, de l'engrais, du fumier, du carburant, des produits phytosanitaires, de la main d'œuvre salariée mais aussi les frais de communication et de transport. Le produit brut ou la recette est obtenu en multipliant la quantité totale de l'extrant par son prix unitaire.

-La marge nette $(\mathrm{MN})$ est la différence entre la marge brute $(\mathrm{MB})$ et les charges fixes, formulée comme suit :

MN = MB - Charges Fixes

Les charges fixes concernent l'amortissement la charrette, la motopompe et les tuyauteries. L'amortissement linéaire se calcule de la manière suivante :

Amorti $=\left(\frac{\mathbf{P m}}{\mathbf{D v}}\right)$

Pm est le prix du matériel et Dv sa durée de vie. 


\subsubsection{Analyse des déterminants de la rentabilité économique}

La théorie économique offre de nombreuses approches pour estimer l'effet d'une ou de plusieurs variables dites explicatives sur une variable dite «expliquée » (Nembot \& Ningaye, 2011). La plus couramment utilisée fait appel à la statistique analytique à travers des modèles de régression (Arshadi \& Lawrence, 1987 ; Bouke, 1989). Dans le cadre de la présente étude, l'approche paramétrique de la frontière de production stochastique a été préférée du fait de certaines réalités du domaine agricole en général et du maraîchage en particulier. En effet, Selon Coelli et al. (1998), les frontières de type stochastique semblent être plus appropriées que la méthode non paramétrique dans le domaine agricole, en particulier pour les pays en développement, où les données sont fortement influencées par des phénomènes aléatoires. Le modèle de la frontière stochastique a été initialement introduit de façon simultanée par Aigner et al. (1976). Dans le secteur agricole, plusieurs chercheurs ont utilisé la fonction Cobb-Douglass pour analyser les déterminants de la rentabilité ou de la performance des exploitations agricoles (Padonou et al., 2011 ; Fontan, 2012 ; Choukou al., 2017).

Pour la simplicité des calculs, la fonction de production CobbDouglass est privilégiée par rapport à la forme translog. La formule générale de la fonction de production Cobb-Douglass se présente comme suit :

$$
\boldsymbol{Q}=\boldsymbol{A} \boldsymbol{K}^{\alpha} \boldsymbol{L}^{\boldsymbol{\beta}}
$$

$\mathrm{Q}$ est le niveau de production ; K, le niveau de capital ; L, niveau de travail ; $\mathrm{A}, \alpha$ et $\beta$ sont les constantes déterminées par la technologie.

Toutes les variables se référant au profil du maraicher, au ménage qu'il prend en charge, aux caractéristiques de son exploitation, à son affiliation et à ses stratégies de commercialisation, ont été testées dans le modèle de régression. Pour éviter les problèmes de multi-colinéarité, certaines variables explicatives présentant une forte corrélation avec d'autres ont été éliminées dans modèle de base. Ainsi, douze (12) variables ont été testées et incluses dans le modèle. La forme empirique de régression se présente comme suit :

$\operatorname{LogMN}=b_{0}+b_{1}$ Age $+b_{2}$ Sexe $+b_{3}$ ExpérienceMS $+b_{4}$ Affiliation + $\mathrm{b}_{5}$ Instruction $+\mathrm{b}_{6}$ Encarement $+\mathrm{b}_{7}$ SupMH $+\mathrm{b}_{8}$ Capital $+\mathrm{b}_{9}$ VGBP $+\mathrm{b}_{10}$ VIM $+b_{11}$ VIB $+b_{12}$ TailleMenage (5).

$b_{0}$ est une constante. Les autres (de $\left.b_{1} a ̀ b_{12}\right)$ sont des coefficients qui vont être déterminés à l'aide de la régression linéaire. MS : maraîchage en campagne sèche, $\mathrm{MH}$ : maraîchage d'hivernage, VGBP : vente groupée bord parcelle, VIM : vente individuelle au marché, VIB : vente individuelle bord-parcelle. 
Tableau 1. Effets attendus des variables explicatives

\begin{tabular}{|c|c|c|c|}
\hline \multicolumn{4}{|c|}{ Variable dépendante : La marge nette (Nette) } \\
\hline $\begin{array}{l}\text { Variables } \\
\text { explicatives }\end{array}$ & Description & $\begin{array}{l}\text { Signe } \\
\text { attendu }\end{array}$ & Modalités \\
\hline Age & Age du chef d'exploitation & + & Variable continue \\
\hline Sexe & Sexe du chef d'exploitation & + & $\begin{array}{l}0=\text { féminin } \\
1=\text { masculin }\end{array}$ \\
\hline Expérience MS & $\begin{array}{l}\text { Son expérience en maraîchage } \\
\text { sec en années }\end{array}$ & + & Variable continue \\
\hline $\begin{array}{ll}\text { Affiliation } & \text { aux } \\
\text { Organisations } & \\
\text { Paysannes (OP) } & \\
\end{array}$ & Appartenance à une OP & + & $0=$ non, $1=$ oui, \\
\hline Instruction & $\begin{array}{l}\text { Niveau d'instruction du chef } \\
\text { d'exploitation }\end{array}$ & + & $0=$ non, $1=$ oui, \\
\hline Capital & Montant investi & + & Continue \\
\hline $\begin{array}{l}\text { Superficie } \\
\text { Maraîchage } \\
\text { d'Hivernage }\end{array}$ & $\begin{array}{l}\text { Sa superficie en } \mathrm{MH} \text { en mètre } \\
\text { carré. }\end{array}$ & + & Variable continue \\
\hline VGBP & Vente groupée bord-parcelle & & $0=$ non, $1=$ oui \\
\hline VIBP & $\begin{array}{lll}\text { Vente } & \text { individuelle } & \text { bord } \\
\text { parcelle } & & \\
\end{array}$ & + & $0=$ non, $1=$ oui \\
\hline VIM & Vente individuelle Marché & $+/-$ & $0=$ non, $1=$ oui \\
\hline Accès encadrement & Accès à la vulgarisation & + & $0=$ non, $1=$ oui \\
\hline Taille ménage & $\begin{array}{l}\text { Nombre de personnes prises en } \\
\text { charge }\end{array}$ & $+/-$ & Variable continue \\
\hline
\end{tabular}

\section{Résultats-Discussion}

\subsection{Caractéristiques socioéconomiques et démographiques}

Le Tableau 2 indique que l'âge moyen des chefs d'exploitation maraîchères d'hivernage est de 47 ans. L'expérience minimale des chefs d'exploitation enquêtés, en maraîchage de la campagne sèche est de 6 ans alors que ce résultat représente la moyenne pour l'expérience en hivernage. Cela constitue un facteur capital dans la bonne conduite et la réussite de toute activité. La pratique du maraîchage d'hivernage est donc récente mais commence à prendre de l'ampleur dans la zone étudiée. En moyenne, les superficies exploitées en maraîchage au cours de la campagne sèche (de novembre à mars) sont 12 fois plus grandes que celles du maraichage d'hivernage (de juin à octobre). Cela pourrait s'expliquer par la « nouveauté » du maraîchage d'hivernage aux yeux des producteurs. Or, en milieu paysan, toute nouveauté fait d'abord l'objet de réticence avant de connaitre une adoption fulgurante. En plus, en saison sèche, des grandes superficies (souvent plus d'un hectare par maraîcher) sont exploitées pour la production de la pomme de terre et du chou dans les deux communes étudiées. Cela dénote les possibilités qu'ont les paysans pour étendre les superficies destinées au maraîchage d'hivernage. 
Le Tableau 2 montre aussi que la taille moyenne des ménages est d'environ 9 personnes pour un nombre d'actifs agricoles mobilisés de 2,37. La comparaison du nombre de personnes en charge par rapport au nombre d'actifs agricoles mobilisés indique que chaque actif agricole mobilisé (1AA) prend en charge environ 4 personnes (Tableau 2).

Tableau 2. Caractéristiques socioéconomiques

\begin{tabular}{|c|c|c|c|c|}
\hline Variables & Minimum & Maximum & Moyenne & Ecart type \\
\hline Age & 25 & 61 & 46,48 & 6,79 \\
\hline Année expérience MS & 6 & 30 & 14,41 & 5,37 \\
\hline Année expérience MH & 3 & 17 & 5,98 & 2,60 \\
\hline Taille du ménage & 4 & 17 & 8,97 & 2,69 \\
\hline $\begin{array}{c}\text { Nombre d'actifs } \\
\text { agricoles }\end{array}$ & 1 & 4,3 & 2,37 & 0,86 \\
\hline Superficie MS & 200 & 15000 & 2140,83 & 2938,35 \\
\hline Superficie MH & 70 & 1500 & 179,96 & 171,10 \\
\hline
\end{tabular}

Il ressort de l'analyse du Tableau 3, que le maraîchage d'hivernage est une activité agricole essentiellement masculine. En effet, 98\% des maraîchers étudiés sont des hommes. En hivernage, les femmes ne se font remarquer que lors des opérations de récolte tandis qu'elles font partie intégrante du maraîchage en saison sèche. Seules deux veuves ont repris l'exploitation maraîchère après le décès de leurs époux. L'accès aux terres est toujours plus facile après le mariage.

Concernant le niveau d'instruction des chefs d'exploitation, il ressort du Tableau 3 qu'environ la moitié des maraîchers interrogés est instruite avec $34,3 \%$ ayant un niveau d'étude compris entre le primaire et le secondaire, $10,8 \%$ ayant fait des études coraniques et $1 \%$ ayant bénéficié de cours d'alphabétisation fonctionnelle. Le niveau d'instruction peut être synonyme d'ouverture à l'innovation et donc à la modernisation des exploitations maraîchères d'hivernage.

L'étude a montré qu'il existe trois (3) modes d'accès à la terre pour la production maraîchère d'hivernage à savoir l'achat, l'héritage et le prêt. 80,4 $\%$ des terres utilisées pour le maraîchage d'hivernage ont été acquises par l'héritage. Après l'héritage, viennent les achats et les prêts avec respectivement des taux de $10,8 \%$ et de $8,8 \%$. L'achat de ces terres est motivé par l'engouement des paysans de la localité mais aussi d'autres contrées pour le maraîchage. Selon les producteurs, ce mode d'accès va concurrencer l'héritage dans les années à venir. En effet, certains producteurs ayant hérité les terres ne sembleraient pas mesurer les opportunités de l'exercice du maraîchage d'hivernage. Ils sont tentés de vendre une partie de leurs terres pour investir dans le commerce. 
Tableau 3. Caractéristiques sociodémographiques

\begin{tabular}{|c|c|c|c|}
\hline Variables & Modalités & Effectif & Proportion \\
\hline \multirow{3}{*}{ Situation matrimoniale } & Marié & 100 & $98,0 \%$ \\
\hline \multirow{3}{*}{ Niveau d'instruction } & Veuve & 2 & $2,0 \%$ \\
\cline { 2 - 4 } & Non scolarisé & 55 & $53,9 \%$ \\
\cline { 2 - 4 } & Primaire & 30 & $29,4 \%$ \\
\cline { 2 - 4 } & Secondaire & 5 & $4,9 \%$ \\
\cline { 2 - 4 } & Coranique & 11 & $10,8 \%$ \\
\cline { 2 - 4 } & Cours d'adultes & 1 & $1,0 \%$ \\
\hline \multirow{2}{*}{ Affiliation aux Organisations } & Oui & 66 & $64,7 \%$ \\
\hline \multirow{2}{*}{ Paysannes } & Non & 36 & $35,3 \%$ \\
\hline \multirow{2}{*}{ Modes d'Acquisition de la terre } & Héritage & 82 & $80,4 \%$ \\
\cline { 2 - 4 } & Achat & 11 & $10,8 \%$ \\
\cline { 2 - 4 } & Prêt & 9 & $8,8 \%$ \\
\hline
\end{tabular}

\subsection{Analyse de la rentabilité économique et financière}

Le Tableau 4 présente la structure du coût de production d'une part et la rentabilité financière des exploitations maraîchères d'hivernage. Sur une superficie moyenne de $180 \mathrm{~m}^{2}$, les coûts variables (60941 FCFA) représentent $82,35 \%$ du coût total de production qui est de $412 \mathrm{FCFA} / \mathrm{m}^{2}$. Dans l'allocation des ressources liées aux coûts variables, les semences et le carburant occupent respectivement les deux premières places $(29,5 \%$ et $33,32 \%)$.

Pour le premier intrant, cela pourrait s'expliquer par la cherté des semences adaptées à la saison mais aussi les pertes de semis enregistrées en pépinière, tandis que pour le second, les maraîchers évoquent le besoin pressant en eau à partir de deux (2) semaines après le repiquage. Ce résultat contredit l'hypothèse selon laquelle le besoin en eau des cultures maraîchères en hivernage serait faible du fait de la pluviosité. Ce résultat est contraire à celui de Savi (2009) pour lequel les produits fertilisants et la main d'œuvre salariée sont les principaux postes de dépenses dans la production de Crincrin (Corchorus olitorius). L'amortissement linéaire a été considéré dans le calcul des coûts fixes. Ces derniers représentent $25,73 \%$ du coût total de production. Le Tableau 4 indique que le produit brut moyen est de 257 134 FCFA. Rapporté au coût total de production, l'on obtient un coefficient multiplicateur moyen de 3,47. La marge brute moyenne est de 196193 FCFA. Les marges nettes minimale (3000 FCFA) et maximale (1 878 300), renseignent sur la différence qui existe entre maraîchers du point de vue de la rentabilité même si tous les maraîchers enquêtés réalisent des résultats positifs. Tout de même, le gain estimé à 177156 FCFA permet de satisfaire les besoins primaires à une période où les ressources financières se font rares et mais aussi de réinvestir dans le maraîchage de la campagne sèche. Ce résultat est similaire aux travaux de Choukou et al. en 2017 sur la production du maïs dans le 
Kanem au Tchad et à ceux de Savi (2009) sur la production du Crincrin (Corchorus olitorius).

Tableau 4. Composantes des charges et produit (en FCFA)

\begin{tabular}{|l|r|r|r|r|}
\hline Rubriques & Minimum & Maximum & Moyenne & Ecart type \\
\hline Semences & 3500 & 87500 & 16255 & 12487 \\
\hline Carburant & 4250 & 70500 & 18314 & 11841 \\
\hline Fertilisant & 0 & 28500 & 6825 & 5207 \\
\hline Produits phyto & 0 & 29500 & 6868 & 6344 \\
\hline Main d'œuvre & 1000 & 64500 & 6236 & 8383 \\
\hline Coût intrants agricoles & 17500 & 242700 & 54972 & 34887 \\
\hline Transport & 0 & 96000 & 4820 & 13830 \\
\hline Communication & 0 & 3500 & 1150 & 702 \\
\hline Coûts variables & 18000 & 340700 & 60941 & 43605 \\
\hline Coûts fixes & 1200 & 29000 & 19037 & 5046 \\
\hline Coût total & 24600 & 258700 & 74000 & 35923 \\
\hline Produit brut & 41300 & 2235000 & 257134 & 251550 \\
\hline Marge brute (MB) & 20800 & 1894300 & 196193 & 214640 \\
\hline Marge Nette (MN) & 3000 & 1878300 & 177156 & 214545 \\
\hline
\end{tabular}

\subsection{Déterminants socioéconomiques et techniques de la rentabilité}

Les résultats du Tableau 5 montre que le modèle d'estimation des déterminants de la rentabilité est globalement significatif $(p<0,001)$ avec un coefficient de détermination multiple ajusté $\mathrm{R}^{2}$ de 0,79 . Ce qui indique que $79 \%$ de la variation totale de la marge nette $(\mathrm{MN})$ est expliqué par les douze variables incluses dans le modèle. Quatre d'entre elles ont présenté un effet significatif à savoir l'expérience, la superficie exploitée, la vente groupée bord parcelle et la vente individuelle au marché. Le coefficient de la variable « Expérience_MS »a été significativement positif $(\mathrm{p}<0,1)$. Cela indique que plus le nombre d'années d'expérience augmente meilleure est la marge nette. L'âge n'affecte pas significativement la marge nette $(\mathrm{p}=0,5063)$. Ces résultats montrent que les maraîchers âgés ne sont pas forcément les plus expérimentés. Ces derniers mettent à profit leur savoir-faire en maraîchage au cours de la campagne sèche pour rentabiliser le maraîchage d'hivernage. Le coefficient de la variable «Sup_MH » a été significativement positif $(\mathrm{p}<0,001)$. Ainsi, plus la superficie exploitée est grande, plus la marge nette croît. Ce résultat est similaire à celui des travaux de Choukou (2017) et Kouakou (2019). Ces travaux ont montré que la rentabilité est significativement corrélée à la superficie emblavée. Ce résultat est néanmoins contraire aux résultats de travaux de Sen (1962), Berry et Cline (1979), et Deolalikar (1981) qui avaient confirmé la thèse de la relation inverse, soutenant que la productivité des petites exploitations est supérieure à celle des grandes. Pour ces auteurs, plus la taille de l'exploitation est grande, plus celle-ci est inefficace. 
En ce qui concerne les trois stratégies de commercialisation testées dans le modèle, la variable "VIM », c'est-à-dire la vente individuelle au marché, influence positivement la rentabilité des cultures maraîchères $(\mathrm{p}=0,0678)$. Ce qui est loin d'être le cas de la variable «VGBP » avec un coefficient négatif. La variable «VIBP » a eu une influence positive mais non significative sur la rentabilité. Ce résultat révèle que les maraîchers qui ciblent et intègrent les marchés bénéficient d'un prix très rémunérateur, réalisent plus de gain que ceux qui restent sur place pour attendre l'arrivée de clients à la parcelle. Le niveau d'instruction, l'accès à l'encadrement et la taille du ménage n'ont pas été significatifs dans le modèle.

Tableau 5. Appréciation des déterminants du modèle

\begin{tabular}{|c|c|r|c|}
\hline & \multicolumn{3}{|c|}{ Variable expliquée : Marge Nette (MN) } \\
\hline \multirow{2}{*}{ Variables explicatives } & Coefficient & Erreur type & $t$ \\
\hline Age & 1117,76 & 1672,65 & 0,67 \\
\hline Sexe & 55448,84 & 71786,91 & 0,77 \\
\hline Année Expérience MH & $2605,51^{*}$ & 4791,08 & 0,54 \\
\hline Affiliation aux OP & $-1288,51$ & 23050,89 & $-0,06$ \\
\hline Instruction & $-15492,05$ & 21913,17 & $-0,71$ \\
\hline Encadrement & $-1204,76$ & 20891,27 & $-0,06$ \\
\hline Superficie exploitée MH & $996,14 * * *$ & 83,47 & 11,93 \\
\hline Capital fixe & $-2,76$ & 2,04 & $-1,35$ \\
\hline VGBP & $-11740,35^{*}$ & 62394,85 & $-0,19$ \\
\hline VIM & $11173,29^{*}$ & 26853,60 & 0,42 \\
\hline VIBP & 6620,92 & 27339,51 & 0,24 \\
\hline Taille du ménage & 893,01 & 4628,46 & 0,19 \\
\hline Constante & $-99701,08^{* * *}$ & 122677,93 & $-0,81$ \\
\hline
\end{tabular}

$\mathrm{N}=102 ; \mathrm{F}(14,87)=29,58 ; \mathrm{R}^{2}$ ajustée $=0,79 ; \mathrm{P}=0,000 ;$

$(*),(* *)$ et $(* * *)$ désignent que le résultat est respectivement significatif au seuil de $10 \%$, de $5 \%$ et de $1 \%$ dans le modèle.

\section{Conclusion}

Au regard de ce qui qui précède, le maraîchage d'hivernage est une activité économique pratiquée majoritairement par les hommes. La plupart d'entre eux est relativement jeune avec un niveau d'instruction plus élevé au regard du niveau général en milieu paysan nigérien. Ce sont des exploitants dotés d'une grande expérience en maraîchage de la campagne sèche, plus ouverts à l'innovation. Le maraîchage d'hivernage est rentable dans les communes de Tagazar et d'Imanan. La marge nette (MN) est positive chez tous les producteurs enquêtés. Plusieurs facteurs socioéconomiques, sociodémographiques et techniques ont influencé soit de façon positive soit de façon négative cet indicateur de la rentabilité. Le test du modèle a été 
significatif et les facteurs introduits ont expliqué à $79 \%$ la variation de la marge nette. Les signes positifs attendus pour le nombre d'années d'expérience et la superficie exploitée ont été confirmés, à l'issue de l'étude. Cependant, l'âge, le sexe, l'accès à l'encadrement, l'affiliation aux OP n'ont pas de relation significative avec la marge nette générée dans la pratique du maraîchage en hivernage. Les Organisations Paysannes et les services techniques publics et privés devraient s'investir plus dans l'encadrement des paysans sur le maraîchage d'hivernage. Ce type de maraîchage est surtout l'activité des paysans les plus entreprenants qui n'attendent jamais un soutien extérieur pour atteindre leurs objectifs.

\section{References:}

1. Aigner, D.-J., Lovell C.-A.-K, \& Schmidt, P. (1976). Formulation and estimation of stochastic frontier production function models. Journal of econometrics, vol. 6, p. 21-38.

2. Alpha, G. \& Dramé, A. (2006). Histoire des crises alimentaires au Sahel : cas du Niger, 71p.

3. Arshadi, N. \& Lawrence, EC. (1987). «An empirical investigation of new bank of performance », Journal of Banking and Finance, Volume 11, P 33-48.

4. Babacar, F., Sadibou, S., Mamadou, D.F., Bachir, W. (2020). Les performances agro-économiques de l'urée:

Doi:10.19044/esj.2020.v16n13p364

URL:http://dx.doi.org/10.19044/esj.2020.v16n13p364 Super Granulé : Cas Du Riz Au Sénégal.

5. Balme, M., Galle, S. \& Thierry, L. (2005). Démarrage de la saison des pluies au Sahel : variabilité aux échelles hydrologique et agronomique, analysée à partir des données EPSAT-Niger. 8p.

6. Bastain, S. \& Fromageot, A. (2007). Le maraîchage : révélateur dynamique des campagnes sahélo-soudaniennes, 19p.

7. Berry, RA. \& Cline, WR. (1979). Agrarian Structure and Productivity in Developing Countries, the Johns Hopkins University Press, Baltimore et Londres.

8. Bourke, P. (1989). « Concentration and other determinants of bank profitability in Europe, North America and Australia », Journal of Banking and Finance, Volume 13, PP 65-79.

9. Butaré, I. \& Zoundi, J. (2005). Eclairer la prise de décision politique en Afrique subsaharienne, 101p.

10. Choukou, MM., Biaou, G., Zannou, A. \& Ahohuendo, B. (2017). Production et rentabilité de la culture de maïs dans les oasis du Kanem au Tchad, 10p Volume 45(3). Published September, 01, 2017 www.jnsciences.org E-ISSN 2286-5314. 
11. Coelli, T., Rao, P. \& Battese, G. (1998). An introduction to efficiency and productivity analysis. Massachusetts, Kluwer Academic Publishers, $275 \mathrm{p}$.

12. Comité Inter Etat de Lutte contre la Sécheresse au Sahel (1982). Développement des cultures pluviales au Niger. 293p.

13. Deolalikar, A. B. (1981). The inverse relationship between productivity and farm size: a test using regional data from India", American Journal of Agricultural Economics, vol. 63 (2), 1981, 275279.

14. Fontan, C. (2012). Production et efficience technique des riziculteurs de Guinée http://journals.openedition.org/economierurale/342 DOI : 10.4000/economierurale.342 ISSN : 2105-2581.

15. Forest, F. \& Cortier, B. (1991). Diagnostic hydrique des cultures et la prévision du rendement régional du mil cultivé dans les pays du CILSS, 11 pages.

16. Hamadou, S. (2000). Evolution à long terme des productions agricoles du système de commercialisation et des prix des produits dans la zone de Maradi, 43p

17. Institut National de la Statistique (2012). Agriculture et conditions de vie des ménages au Niger, 72p.

18. Kouakou, A.M (2019). Rentabilité de la production et commercialisation de l'igname (Dioscorea) en Côte d'Ivoire, p51-56

19. Mahamadou, I., Joachim, B., \& Kabirou Sani, OB. (2018). Analyse des contraintes foncières et culturales du maraîchage dans le département de Kantché au Niger: cas du périmètre irrigué de Tassaou. Doi : 10.19044/esj.2018.v14n30p240

URL:http://dx.doi.org/10.19044/esj.2018.v14n30p240

20. Ministère de l'Agriculture et de l'Elevage (2015). Stratégies de la petite irrigation du Niger. Niamey, éditions Ministère de l'Agriculture. $55 \mathrm{p}$.

21. Nazoumou, Y., Favreau, G., Adamou, MM., \& Maïnassara, I. (2016). La petite irrigation par les eaux souterraines, une solution durable contre la pauvreté et les crises alimentaires au Niger ? Cah. Agric. 25 : 15003. 114p.

22. Nembot, L. \& Ningaye, N. (2011). Réformes financières et rentabilité du système bancaire des États de la CEMAC, DOI: 10.3917/med.155.0111.

23. Padonou, HM. (2011). Analyse socio-économique des systèmes de production agricole à base d'igname dans la commune de Glazoué au Bénin : cas du village Magoumi, 65p. 
24. Papy, B.B. \& Alain Roddy, MK. (2020). Performances Des «Fermes Modèles » Du Corridor De Monkoto Dans La Province De La Tshuapa, République Démocratique Du Congo. Doi:10.19044/esj.2020.v16n24p206

URL:http://dx.doi.org/10.19044/esj.2020.v16n24p206

25. Paul-Alfred, K. (2019): Déterminants de la productivité, de la rentabilité économique et impact social du maraîchage dans la commune de Boundiali, au Nord de la Côte d'Ivoire. Rev. Mar. Sci. Agron. Vét. (2020) 8(1): 93-102.

26. Pirou, JP. (2005). Mesure de la rentabilité des entreprises, 25p

27. Sadi, S.M., Saidou, A.K., Boubé, M. \& Aune, J.B. (2020). Effet de la fertilisation à base de biomasse de Sida cordifolia L. sur les performances Agronomiques et la rentabilité économique de la tomate (Lycopersicum esculentum Mill) en culture irriguée. European $\begin{array}{lllll}\text { Scientific Journal, } & \text { ESJ, } & 16 & \text { (3), } & 127 .\end{array}$ https://doi.org/10.19044/esj.2020.v16n3p127

28. Savi, A.D. (2009). Analyse de la rentabilité financière et de l'efficacité économique de la production du crincrin (Corchorus olitorius) dans la vallée du Mono. Mémoire du diplôme d'études approfondies (DEA), 23-45p.

29. Sen, A.K. (1962). An aspect of Indian agriculture. Economic WeekIy, February, 243-246. 